\title{
Extraordinary Light-Induced Local Angular Momentum near Metallic Nanoparticles
}

\author{
Alessandro Alabastri, ${ }^{*}, \dagger, \ddagger, \Phi \mathrm{I}$ Xiao Yang, ${ }^{\dagger, \ddagger, I L}$ Alejandro Manjavacas, ${ }^{\S}$ Henry O. Everitt, ${ }^{*}, \|, \perp$ \\ and Peter Nordlander*, ${ }^{\dagger}$, \\ ${ }^{\dagger}$ Department of Physics and Astronomy MS 61 and ${ }^{\ddagger}$ Laboratory for Nanophotonics, Smalley-Curl Institute, \\ Rice University, Houston, Texas 77005, United States \\ ${ }^{\S}$ Department of Physics and Astronomy, University of New Mexico, Albuquerque, New Mexico 87131, \\ United States \\ "Army Aviation and Missile RD\&E Center at Redstone Arsenal, Huntsville, Alabama 35898, United States \\ ${ }^{\perp}$ Department of Physics, Duke University, Durham, North Carolina 27708, United States
}

ABSTRACT: The intense local field induced near metallic nanostructures provides strong enhancements for surfaceenhanced spectroscopies, a major focus of plasmonics research over the past decade. Here we consider that plasmonic nanoparticles can also induce remarkably large electromagnetic field gradients near their surfaces. Sizeable field gradients can excite dipole-forbidden transitions in nearby atoms or molecules and provide unique spectroscopic fingerprinting for chemical and bimolecular sensing. Specifically, we investigate how the local field gradients near metallic nanostructures depend on geometry, polarization, and wavelength. We introduce the concept of the local angular

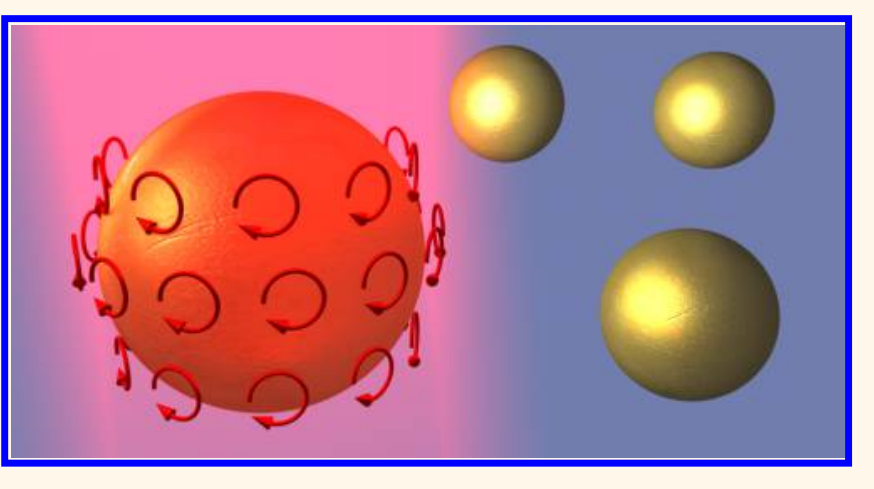
momentum (LAM) vector as a useful figure of merit for the design of nanostructures that provide large field gradients. This quantity, based on integrated fields rather than field gradients, is particularly well-suited for optimization using numerical grid-based full wave electromagnetic simulations. The LAM vector has a more compact structure than the gradient matrix and can be straightforwardly associated with the angular momentum of the electromagnetic field incident on the plasmonic structures.

KEYWORDS: optical angular momentum, local angular momentum, electric field gradient, dipole-forbidden transitions, quadrupolar transitions, plasmonic nanoparticles

\begin{abstract}
S urface plasmons-the collective electromagnetic excitation of conduction electrons in a metal-have been studied extensively over the past decade, primarily because of their ability to induce large local electric field "hot spots" near the surface of metallic nanoparticles (NPs). The intense local electric fields generated by the excitation of these surface plasmon modes have been exploited for a variety of applications, including surface-enhanced spectroscopies, ${ }^{1-6}$ molecular sensing, ${ }^{7-9}$ photovoltaics, ${ }^{10-14}$ photocatalysis, ${ }^{15-19}$ biomedical analyses, ${ }^{20-27}$ and many other nanophotonic applications. $^{28-30}$ Surface plasmon modes confine these electric fields to subwavelength regions through the ability of metallic NPs to couple propagating electromagnetic fields to bound electronic oscillations. This collective electronic motion is restricted to the NP surface, where electrons accumulate and induce remarkably large charge densities. Thus, in addition to enhanced local electric field intensity, dramatically enhanced electric field gradients emerge as the proximal evanescent fields rapidly decay away from the surface within a few nanometers.
\end{abstract}

To date, few applications of this strong local field gradient have been explored, although several preliminary studies have considered using them to detect dipole-forbidden molecular transitions. $^{31-38}$

The connection between electric field gradients and dipoleforbidden transitions becomes apparent when considering the quantum mechanical transition rate between atomic or molecular eigenstates $i$ and $f$ separated by energy $E_{i f}=\hbar \omega_{i f}$. When the system interacts with a perturbing external field, the transition rate between $i$ and $f$ can be expressed through Fermi's golden rule as ${ }^{39}$

$$
\Gamma_{i f}=\frac{2 \pi}{\hbar^{2}}\left|\left\langle i\left|H_{\mathrm{int}}\right| f\right\rangle\right|^{2}\left\{\delta\left(\omega_{i f}-\omega\right)+\delta\left(\omega_{i f}+\omega\right)\right\}
$$

Received: March 16, 2016

Accepted: April 5, 2016 
where $H_{\text {int }}$ is the Hamiltonian that quantifies the interaction between the system and the external field. For a single electron, this Hamiltonian may be written as ${ }^{33,40}$

$$
H_{\mathrm{int}, e}=-\frac{e}{m} \mathbf{p} \cdot \mathbf{A}+\frac{e^{2}}{2 m}|\mathbf{A}|^{2}+e \phi
$$

for charge $e$, canonical momentum $\mathbf{p}$, field vector $\mathbf{A}$, and the scalar field potential $\phi$. When dealing with charge distributions typical of atoms and molecules, it is often more convenient to rewrite the Hamiltonian using a multipolar expansion of the fields, and choosing the appropriate gauge ${ }^{40}$ leads to

$$
H_{\text {int }}=\boldsymbol{\mu} \cdot \mathbf{E}_{0}-\mathbf{Q}:(\nabla \mathbf{E})_{0}-\mathbf{m} \cdot \mathbf{B}_{0}+\cdots
$$

Here, $\boldsymbol{\mu}=\int \mathbf{r} \rho(\mathbf{r}) \mathrm{d}^{3} r, \mathbf{Q}=\mathrm{Q}_{\alpha \beta}=\int\left(3 r_{\alpha} r_{\beta}-r^{2}\right) \rho(\mathbf{r}) \mathrm{d}^{3} r$, and $\mathbf{m}=(1 / 2) \int \mathbf{r} \times \mathbf{J}(\mathbf{r}) \mathrm{d}^{3} r$ are the electric dipole, the electric quadrupole, and the magnetic dipole transition moments, respectively, associated with the charge $\rho(\mathbf{r})$ and current densities $\mathbf{J}(\mathbf{r})$ of the system. ${ }^{41}$

The relative magnitudes of the terms in eq 3 usually decrease with expansion order. In molecules with a permanent dipole moment, the first term will dominate, and the higher-order terms can be neglected. However, many molecules have no dipole moment and do not exhibit dipolar transitions, ${ }^{42}$ such as the homonuclear diatomic molecules $\left(\mathrm{H}_{2}\right)$, oxygen $\left(\mathrm{O}_{2}\right)$, and nitrogen $\left(\mathrm{N}_{2}\right)$. Larger molecules like carbon dioxide $\left(\mathrm{CO}_{2}\right)$, methane $\left(\mathrm{CH}_{4}\right)$, and benzene $\left(\mathrm{C}_{6} \mathrm{H}_{6}\right)$ also possess no dipole moment because of their symmetrical geometry. The optical detection of these types of molecules is exceedingly difficult because their response to electromagnetic radiation is very weak. In most cases, the quadrupolar term of eq 3 is the leading nonzero multipolar term, and quadrupolar transitions have been observed in some cases when the quadrupole moment of the molecule is large and the path length is very long. ${ }^{43-46}$ In the Methods section, we provide explicit expressions for the electric dipolar and quadrupolar transition rates, showing how they can be expressed in terms of the gradient matrix of the electric field.

To understand why it is difficult to induce quadrupolar transitions using standard optical sources, consider the amplitude of the field gradient of a plane wave (PW), given by

$$
\left|\nabla \mathbf{E}_{\mathrm{PW}}\right|=k E_{0}
$$

where $\mathbf{E}_{\mathrm{PW}}=\mathbf{E}_{0} e^{-i \mathbf{k} \cdot \mathbf{r}} e^{-i \omega t}$ is the associated field. Clearly, the gradient of the PW is in the direction of wave propagation, and its magnitude is proportional to the wave vector modulus $k=2 \pi / \lambda$. Since the gradient "sensed" by a molecule of length $a$ is proportional to $k a=2 \pi a / \lambda$, a molecule senses virtually no gradient and, therefore, no quadrupole transition, unless $\lambda \lesssim a^{47,48}$ However, because metallic NPs produce strong local field gradients, molecules near a NP surface may experience a sufficient gradient to induce a quadrupolar transition. In particular, the electric field gradient outside a spherical metallic NP of diameter $d \ll \lambda$ interacting with a plane wave of amplitude $E_{0}$ can be approximated as

$$
|\nabla \mathbf{E}|_{\mathrm{NP}} \sim \frac{E_{0}}{r}\left(\frac{d}{r}\right)^{3}
$$

where $r$ is the distance from the center of the NP. Thus, a molecule near the NP surface will "sense" a gradient proportional to $a / d$, not $a / \lambda$. Because the quadrupolar transition rates depend on the square of the gradient, we expect the NP gradient to enhance the quadrupolar transition rate by

$$
\eta_{\mathrm{Q}}=\frac{\Gamma_{\mathrm{Q}, \mathrm{NP}}}{\Gamma_{\mathrm{Q}, \mathrm{PW}}} \sim\left(\frac{\lambda}{d}\right)^{2}
$$

with respect to the PW gradient. In the Methods section, we provide a detailed derivation of eqs 5 and 6 .

Rotational transitions occur at much larger wavelengths than electronic or vibrational transitions, typically in the millimeter or sub-millimeter region, so the enhancement factor $\eta_{\mathrm{Q}}$ can be enormous. Consider, for example, that NPs with a diameter of $10 \mathrm{~nm}$ can enhance vibrational transition rates by $\sim 10^{6}(\lambda=10 \mu \mathrm{m})$ but rotational transitions by $\sim 10^{10}(\lambda=1 \mathrm{~mm})$. Therefore, quadrupolar transitions are much more favorable for molecules near the surface of metallic nanoparticles, assuming that energy and angular momentum are also conserved.

Similar arguments indicate even stronger enhancement factors for higher multipolar moments since higher-order transitions depend on higher-order derivatives of the fields. A PW has electric field gradients which scale as $k^{n} \sim\left(\frac{1}{\lambda}\right)^{n}$, with $n$ being the derivative order $(n=0$ for dipole, $n=1$ for quadrupole, $n=2$ for octupole, etc.), while the field proximal to the surface of a metallic NP of size $d$ scales as $\left(\frac{1}{d}\right)^{n}$. Consequently, the NP-induced transition rate enhancement factor $\eta_{n}$ is

$$
\eta_{n}=\frac{\Gamma_{n, \mathrm{NP}}}{\Gamma_{n, \mathrm{PW}}} \sim\left(\frac{\lambda}{d}\right)^{2 n}
$$

relative to the $n$-th derivative order, PW-induced transition. In the Methods section, we provide more details regarding the derivation of eq 7. For now, it is important to note that this enhancement does not depend on the excitation of a localized surface plasmon resonance (LSPR) or on the nonradiative losses of the material but only on the lightning rod metallic screening effect of the NP, which extends to long wavelengths $\left(\lambda \gg \lambda_{\text {LSPR }}\right)$. Therefore, the enhancement is relatively independent of the metal used, provided it contains sufficient conduction electron density. However, the excitation of a LSPR provides additional enhancements, as will be shown below. For such excitations, the enhancements will depend on the nonradiative losses in the same manner as the field enhancements: decreasing with increasing loss.

Complex metallic geometries possessing sharp edges where charges can accumulate (e.g., ellipsoids, polyhedra, dimers, cones, branched structures) will produce even larger electric field gradient enhancements than spherical NPs. In most of these cases, the calculations of the induced near-field gradient cannot be performed analytically, so numerical approaches must be used, such as those based on spatial grid discretization (e.g., finitedifference time domain (FDTD) or finite element method (FEM)). Unfortunately, spatial discretization produces some difficulties when calculating the field gradients: they can exhibit significant discontinuities across the grid, making it very difficult to analyze and map the spatial distribution of the field, especially near surfaces. Moreover, the gradient matrix contains nine components that have no direct relationship to specific multipolar moments since the associated transition rates typically depend on linear combinations of the individual matrix components. Consequently, the rational design of nanostructures for enhanced multipolar transition rates would greatly benefit 
from the introduction of a figure of merit whose values would indicate the presence of large gradients.

Here we propose such a quantity, the local angular momentum (LAM), that we define as

$$
\operatorname{LAM}(\mathbf{r})=\frac{1}{V} \varepsilon \iiint_{V} \mathrm{~d} V\left\{\left(\mathbf{r}^{\prime}-\mathbf{r}\right) \times\left[\mathbf{E}\left(\mathbf{r}^{\prime}\right) \times \mathbf{B}\left(\mathbf{r}^{\prime}\right)\right]\right\}
$$

where $V$ is the volume of a small sphere of radius $R_{\mathrm{S}}$ centered around a point $\mathbf{r}$ outside the NP, and $\varepsilon$ is the permittivity of the medium. Its dimensional unit $\left[\mathrm{N} \cdot \mathrm{m} \cdot \mathrm{s} / \mathrm{m}^{3}\right]$ is equivalent to an angular momentum (AM) density since it is derived from the definition of electromagnetic field $\mathrm{AM} .^{41,49,50}$ By choosing a finite volume over which the LAM is defined, we obtain an appreciable advantage because LAM allows us to match the integration sphere and molecular size so the relevant interacting AM may be estimated. In addition, it is computationally advantageous to calculate integral quantities instead of differential ones. Physically, we expect that the transition rates will only be influenced by field gradients within the spatial domain of the molecule (i.e., of length $a=2 R_{S}$ located at $\mathbf{r}$ ). In the Methods section we provide more details about the significance of this integration radius on the LAM calculation.

The connection between the LAM and the field gradients can be made explicit by performing a Taylor series expansion of the fields around $\mathbf{r}$ (see Methods):

$$
\left(\begin{array}{c}
\operatorname{LAM}_{x} \\
\operatorname{LAM}_{y} \\
\operatorname{LAM}_{z}
\end{array}\right) \approx \frac{1}{V} \varepsilon \mu \sum\left(\begin{array}{c}
r_{y}^{2} E_{x y} \\
-r z^{2} E_{z z}-r_{x}^{2} E_{x x} \\
r_{y}^{2} E_{z y}
\end{array}\right) H_{y}
$$

where $E_{i j}=\frac{\partial E_{i}}{\partial j}$ are the Cartesian gradients of the fields, and $\varepsilon$ and $\mu$ are the electric permittivity and magnetic permeability of the ambient medium, respectively. Despite showing only four electric field gradient components, the symmetry of eq 9 actually captures seven of the nine elements of the $\nabla \mathbf{E}$ tensor. The remaining two components $\left(E_{x z}=E_{z x}\right)$ refer to the EM field gradient in the direction of propagation, for which the AM is exactly zero for a PW.

This argument appears paradoxical: a plane wave carries no total AM, but circularly polarized PWs have been proven to transfer a net amount of AM. ${ }^{51}$ This apparent contradiction is solved if one considers real, finite PWs instead of ideal, infinite PWs. For example, the AM carried by a truncated circularly polarized PW is not zero, and the spin angular momentum (SAM) contribution is recovered. ${ }^{52-54}$ Indeed, several studies have investigated the roles played by SAM and orbital angular momentum (OAM) in tailored electromagnetic fields, ${ }^{55-58}$ how they can be generated, ${ }^{59}$ how they couple to a medium, ${ }^{60-63}$ and their implications in multichannel radio transmissions. ${ }^{64,65}$ In the following, different geometries will be analyzed, and it will be shown how the two missing components of eq 9 become dominant only far from the structure, where the field gradient is comparable to the PW background. The key point is that within the integration region, if one component of the gradient is large, the LAM is also large, so the LAM figure of merit can be used to identify regions where enhanced multipolar transition rates can occur. Although single metallic structures will be analyzed here, we note that electromagnetic field shaping has been obtained by employing $2 \mathrm{D}^{59,66}$ and $3 \mathrm{D}^{67}$ periodic or quasi-periodic arrays, ${ }^{66,67}$ and collective excitations ${ }^{68}$ and selective transmission resonances ${ }^{69}$ have been achieved. We believe the LAM concept can be fully extended to these $2 \mathrm{D}$ and $3 \mathrm{D}$ systems, where particle interactions may permit further tuning of the electric field gradient.

\section{RESULTS AND DISCUSSION}

We start by examining the near-fields around a gold sphere modeled using the Johnson and Christy permittivity. ${ }^{70}$ For convenience, we normalize all calculations to the equivalent $\mathrm{LAM}_{0}$ value obtained by employing the electric field gradient of a plane wave $\left(E_{0}=1 \mathrm{~V} / \mathrm{m}\right.$ and wavelength $\left.\lambda\right)$ as the quantity to be integrated into the $R_{\mathrm{S}}$ radius sphere. From eqs 4 and 9, we can derive this quantity as $\mathrm{LAM}_{0}=2 \pi\left|E_{0}\right|^{2} \varepsilon_{0} R_{\mathrm{S}}{ }^{2} / c \lambda$. This normalization will help us quantitatively assess how nanostructures enhance local angular momentum compared with the plane wave case. As shown in Figure 1, a $d=70 \mathrm{~nm}$ sphere is

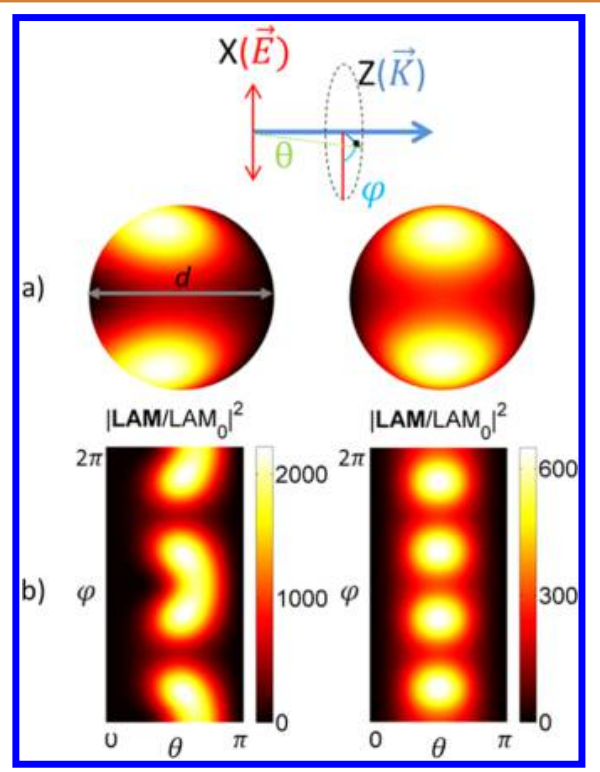

Figure 1. Normalized local angular momentum $|L A M|^{2}$ for a gold sphere with $R_{S}=1 \mathrm{~nm}$ integration radius. (a) LAM distribution $5 \mathrm{~nm}$ away from a $d=70 \mathrm{~nm}$ gold sphere at resonance $(520 \mathrm{~nm})$ (left) and in the long wavelength regime (right). The incident light is propagating along the $z$-axis and is polarized along the $x$-axis. (b) Local angular momentum distribution in the $\theta \varphi$ plane at resonance (left) and in the long wavelength regime (right). LAM values are normalized to $\mathrm{LAM}_{0}=2 \pi \mid E_{0}{ }^{2} \varepsilon_{0} R_{\mathrm{S}}{ }^{2} / c \lambda$.

illuminated by a PW at its LSPR wavelength $\left(\lambda_{\mathrm{LSPR}}=520 \mathrm{~nm}\right.$, left) and at a wavelength far from resonance ( $5 \mu \mathrm{m}$, right). Figure 1a shows the LAM distribution $5 \mathrm{~nm}$ away from the sphere surface, whereas Figure $1 \mathrm{~b}$ plots the LAM distribution in spherical coordinates. The calculations are performed using Mie theory with an integration radius $R_{\mathrm{S}}=1 \mathrm{~nm}$. Compared to a linearly polarized $\mathrm{PW}$ with vanishing $\mathrm{AM}^{53}$ the presence of a metallic sphere clearly generates a large LAM around its surface. Interestingly, both the magnitude and spatial distributions of the LAM are similar for resonant and nonresonant wavelengths. For nonresonant excitation, the electric field distribution is determined by the lightning rod effect and remains virtually unchanged at wavelengths beyond $5 \mu \mathrm{m}$. In the following, we will refer to this as the long wavelength limit. By contrast, the LAM at the LSPR is enhanced, but only modestly so (approximately three times larger) and only over a narrow spectral window. 

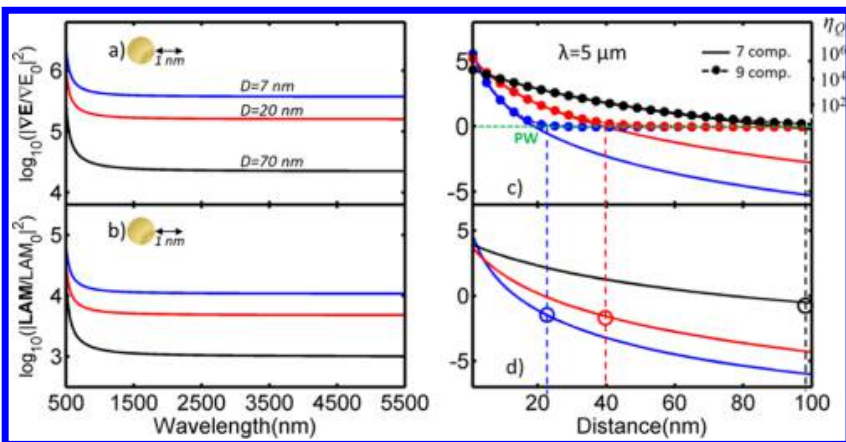

Figure 2. Left: Wavelength dependence of the total electric field gradient $[\mathbf{V} /(\mathbf{m} \cdot \mathbf{n m})](\mathrm{a})$ and normalized local angular momentum (b) at $1 \mathrm{~nm}$ above the surface of Au spheres with diameters of $7 \mathrm{~nm}$ (blue), $20 \mathrm{~nm}$ (red), and $70 \mathrm{~nm}$ (black). The radius of the integration sphere $R_{\mathrm{S}}=0.1 \mathrm{~nm}$. Right (same units as left): (c) Distance dependence from the sphere surface of the 7 component ( 7 comp.) partial electric field gradient (solid lines) and the 9 component $(9$ comp.) total electric field gradient (dotted lines) for the same NP diameters. The total gradient for a plane wave $(\lambda=5 \mu \mathrm{m})$ is also reported (green dashed line). Vertical dashed lines indicate the distance at which total and partial gradients begin to diverge. (d) Distance dependence of LAM for the same NP diameters. Circles indicate the maximum distance at which LAM accurately follows the total gradient, and the gradients are enhanced compared to a PW. LAM values are normalized to $\operatorname{LAM}_{0}=2 \pi\left|E_{0}\right|^{2} \varepsilon_{0} R_{\mathrm{S}}^{2} / c \lambda$.

To explore how the LAM magnitude is related to gradient intensity, Figure 2 plots $|\nabla E|^{2}$ and $|L A M|^{2}$ over a wide wavelength range (Figure 2a,b) and at increasing distances from the NP (Figure 2c,d). NPs with three different diameters ( $d=7 \mathrm{~nm}$ (blue), $20 \mathrm{~nm}$ (red), and $70 \mathrm{~nm}$ (black)) are compared using an integration sphere with $R_{\mathrm{S}}=0.1 \mathrm{~nm}$. In panels a and $b$, both the gradient and the LAM are calculated $1 \mathrm{~nm}$ away from the sphere surface, and their magnitude decreases with increasing wavelength away from resonance, quickly reaching a finite constant value. This property is crucial for enhancing molecular transition rates: even in the long wavelength regime where rovibrational transitions occur, metal NPs still produce large electric field gradients and thereby enhance multipolar transition rates for molecules therein.

Figure $2 \mathrm{c}, \mathrm{d}$ analyzes how the gradient and the LAM decrease as the integration region moves away from the NP surface. In Figure 2c, we plot the partial gradient magnitude as a function of distance from the NP (solid lines), calculated by including only the seven components shared with the LAM, while the dotted lines include all nine components of the gradient. Comparison of Figure $2 \mathrm{c}, \mathrm{d}$ in the near-field region reveals excellent qualitative agreement between the seven-component gradient and the LAM, as expected from eq 9. The agreement ends at the horizontal green dashed line that represents the PW gradient in the long wavelength limit $(\lambda=5 \mu \mathrm{m})$. Here, total gradient departs from the partial gradient and from the LAM, but this is an uninteresting region where $\left|\nabla E_{\mathrm{NP}}\right|^{2} \cong\left|\nabla E_{\mathrm{PW}}\right|^{2}$ and the dominant $\mathrm{AM}$ contribution comes from the $\mathrm{PW}$, so $\eta \cong 1$, and the NP does not provide any enhancement. This analysis clearly supports LAM as a convenient figure of merit in the search for spatial regions with large field gradients.

Note that the interesting near-field region, where gradient enhancement is strongest, extends to the vertical dashed lines and expands with increasing NP diameter $d$. Although the larger NPs therefore have a larger volume of enhanced gradients, the curves cross close to the NP surface, and the highest gradients and LAM are associated with the smallest NPs, just as eqs 6 and 7 predicted. Figure $2 \mathrm{~d}$ shows how LAM rapidly increases toward the surface where $\left|\nabla E_{\mathrm{NP}}\right|^{2} \gg\left|\nabla E_{\mathrm{PW}}\right|^{2}$, increasing up to 6 orders of magnitude for the smallest NP relative to the $\eta \cong 1 \mathrm{PW}$ value.

The results shown in Figure 1 and Figure 2 were obtained using an analytical approach based on Mie theory. However, more complicated nanostructures require the use of numerical simulation tools like the FDTD method, and concerns about grid discretization must be addressed. In the Methods section we demonstrate the robustness of the FDTD calculations by comparing them with Mie theory and FEM results.

To investigate the influence of the nanoparticle shape and structure for the LAM enhancements, we introduce the parameter $\gamma_{i j}=\left.\frac{\max L^{\mathrm{AM}}}{L^{\mathrm{AM}}}\right|_{w}$, defined as the ratio between the maximum and mean ${ }^{w}$ LAM values calculated on a rectangular perimeter in the $i j$ plane at a distance $w$ from the nanostructure. (We set $w=4 \mathrm{~nm}$ in this paper.) The purpose of this parameter is to locate regions with large LAM, and the nanostructure geometry will guide which planes to choose. For example, symmetry planes are a natural choice for symmetric nanostructures, while nonsymmetric structures will require a larger number of planes to sample the LAM pattern.

The $\gamma_{i j}$ parameter is useful to characterize different geometries and provides insights about geometry optimization. For example, in spectroscopic measurements with large LAM but randomly positioned and oriented molecules, a value of $\gamma \approx 1$ is desired so that the nanostructures do not need to be precisely located. On the other hand, $\gamma \gg 1$ can be required whenever strongly localized LAM enhancement is needed. The $\gamma_{i j}$ quantity is particularly useful for mapping the LAM distribution outside nanostructures of complex shapes. In the long wavelength regime, the spherical case analyzed above produces $\gamma_{x y}=1.8$ and $\gamma_{x z}=4.4$, showing that LAM is relatively homogeneously distributed in the $x y$ plane.

Moving to more complex systems, we analyze the case of a dimer composed of two identical $d=70 \mathrm{~nm}$ Au spheres separated by a $10 \mathrm{~nm}$ gap. The corresponding LAM and field gradients are shown in Figure 3. As for the individual sphere, the spatial dependences of the dimer LAM and gradient are very similar: the enhancements can be very large near the NP surfaces, especially in the gap region. A similar pattern was found for the quadrupolar field enhancements of a sphere dimer by Filter et al., 32 confirming that LAM serves as a good indicator of electric field gradient intensity. NP dimers are widely used to enhance dipole-allowed transitions that depend on field intensity. ${ }^{2,4}$ Although the magnitude of the field enhancement in the gap is very large, it is interesting to note that the LAM is very small at the gap center, where the spatial distribution is homogeneous and gradients are weak. Nevertheless, $\gamma_{x y}=7.1 \approx \gamma_{x z}=6.9$ despite the lack of a clear hot spot in the $x z$ plane (Figure $3 \mathrm{~d}$ ). This is due to the relatively inhomogeneous pattern of the LAM and gradient, whose absolute values are generally low.

More complex nanostructures generate even more inhomogeneous near-fields and are expected to produce even larger LAM enhancements. Figure 4 compares the LAM $(\mathrm{a}, \mathrm{c})$ and the gradient $(b, d)$ intensities for a gold cone $(70 \mathrm{~nm}$ wide, $100 \mathrm{~nm}$ high, $3.5 \mathrm{~nm}$ tip radius) illuminated by a plane wave propagating perpendicularly to the cone axis. The LAM is calculated using FDTD with a $0.25 \mathrm{~nm}$ mesh size and an integration radius $R_{S}=1 \mathrm{~nm}$. The side views in the long wavelength limit $(\lambda=5 \mu \mathrm{m})$ (Figure $4 \mathrm{c}, \mathrm{d})$ demonstrate that 


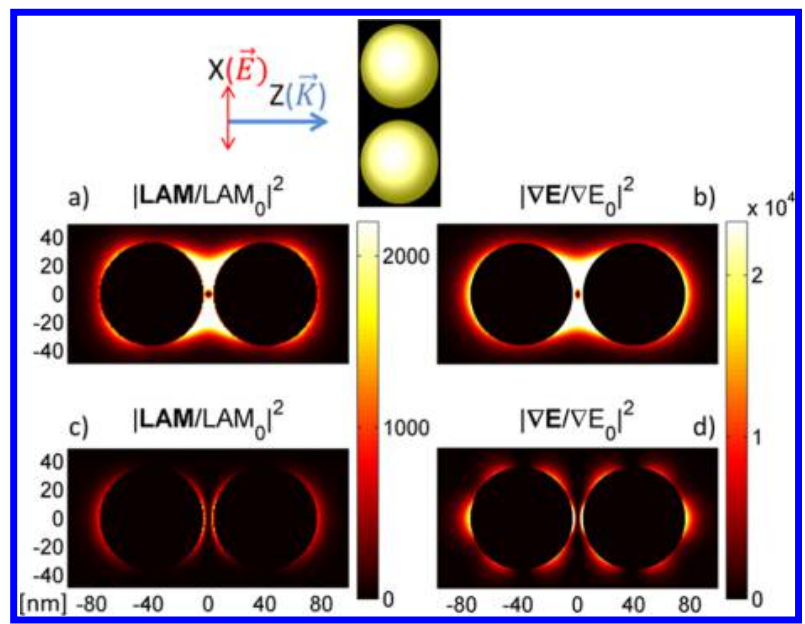

Figure 3. Linearly polarized plane wave at $5 \mu \mathrm{m}$ propagates along $Z$ and interacts with a gold sphere dimer (spheres are $70 \mathrm{~nm}$ wide with a $10 \mathrm{~nm}$ gap). (a) Top view of the normalized LAM intensity ( $x y$ plane). (b) Top view of the gradient intensity ( $x y$ plane). (c) Side view of the normalized LAM intensity ( $x z$ plane). (d) Side view of the gradient intensity ( $x z$ plane). The grid size in the FDTD simulations is $0.25 \mathrm{~nm}$. Vertical and horizontal axes represent distances in nanometers. LAM values are normalized to $\mathrm{LAM}_{0}=2 \pi \mid$ $\left.E_{0}\right|^{2} \varepsilon_{0} R_{S}^{2} / c \lambda$.

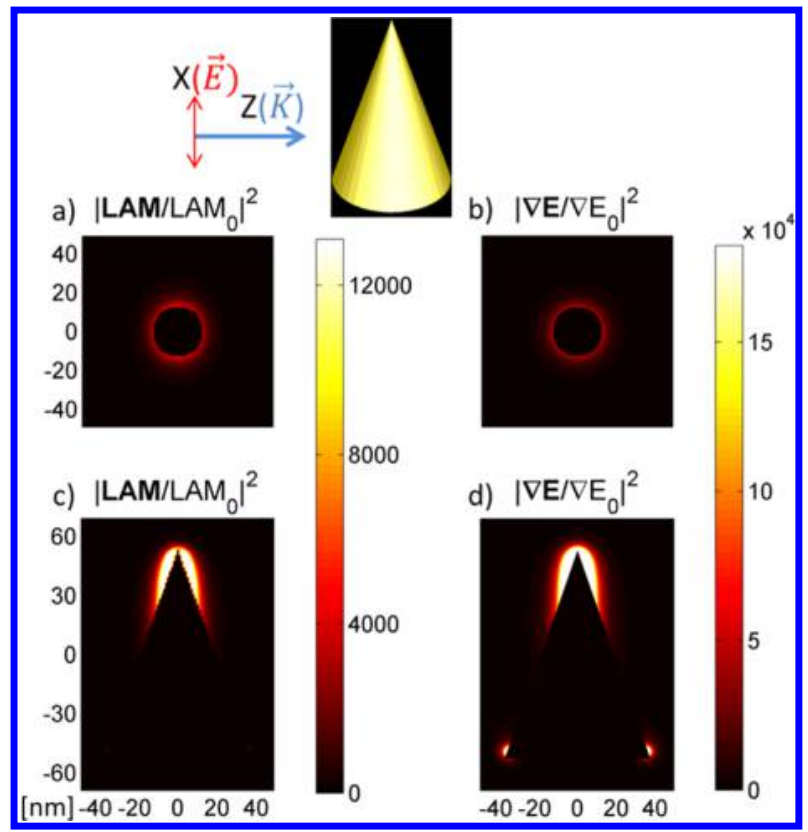

Figure 4. Linearly polarized plane wave along $X$ at $5 \mu \mathrm{m}$ propagates along $Z$ and interacts with a gold cone $(70 \mathrm{~nm}$ wide and $100 \mathrm{~nm}$ high with a $3.5 \mathrm{~nm}$ tip radius) in the long wavelength limit $(5 \mu \mathrm{m})$. (a) Top view of the normalized LAM radial intensity ( $y z$ plane). (b) Top view of the gradient radial intensity ( $y z$ plane). (c) Side view of the LAM vertical intensity ( $x z$ plane). (d) Side view of the gradient vertical intensity ( $x z$ plane). The grid size in the FDTD simulations was $0.25 \mathrm{~nm}$. Vertical and horizontal axes represent distances in nanometers. $L A M$ values are normalized to $\mathrm{LAM}_{0}=2 \pi \mid$ $E_{0} I^{2} \varepsilon_{0} R_{S}^{2} / c \lambda$.

both the LAM and the field gradient are significantly enhanced near the tip of the cone. Moreover, the LAM is enhanced around the circumference of the cone, with a radial profile resembling the gradient contour (Figure 4a,b). Notice that the gradient enhancement is stronger around the base of the structure (Figure 4d) than the LAM (Figure 4c) because the $E_{x z}=E_{z x}$ components are not included. In the Methods section we furnish quantitative details about this marginal disagreement. Nevertheless, the majority of the signal comes from the tip region where LAM nicely captures the field gradient behavior.

As expected, structures exhibiting sharp regions lead to highly inhomogeneous LAM and gradient distributions as charges efficiently accumulate in small regions and produce large electric fields and gradients. This asymmetry is well captured by the $\gamma_{i j}$ parameters for the cone, $\gamma_{y z}=1.92$ and $\gamma_{x z}=35.4$, although $\gamma_{x z} \approx \gamma_{x y}$ due to the symmetry about the $X$ axis.

We now turn to an even more complex nanostructure, a gold octopod (Figure 5a), whose low symmetry makes the connection between the three components of LAM and the nine components of the electric field gradient even more explicit. The octopod has eight branches-four in the $x y$ plane and four in the $x z$ plane-that can be thought of as a sphere surrounded by radially directed cones, a combination of the previously investigated geometries. As before, the structure is irradiated with a plane wave propagating in the $z$-direction, polarized in the $x$-direction, in the long wavelength limit $(\lambda=5 \mu \mathrm{m})$, as shown in Figure 5a. The branch length of the octopod is $30 \mathrm{~nm}$, and the angle between adjacent feet is $45^{\circ}$. We calculate the different components of the LAM and the field gradient using the FDTD method with a $0.25 \mathrm{~nm}$ mesh size and a $R_{\mathrm{S}}=1 \mathrm{~nm}$ integration sphere.

Figure $5 \mathrm{~b}, \mathrm{~d}$ shows all nine components of the electric field gradient matrix $E_{i j}=\frac{\partial E_{i}}{\partial x_{j}}$ in the $x y$ and $x z$ planes, respectively, while Figure $5 c$,e contains the corresponding LAM components. In the $x y$ plane, $\mathrm{LAM}_{x}$ and $\mathrm{LAM}_{y}$ are the dominant LAM components, appearing very similar to the respective $E_{x y}$ and $E_{y y}$ components of the gradient matrix. The solid colored squares highlight this connection, as predicted by eq 9, while dashed colored squares indicate which gradient components can be derived from the corresponding solid colored squares using symmetry arguments. A similar correspondence can also be found in the $x z$ plane: here, $\mathrm{LAM}_{y}$ is the dominant component and exhibits the same pattern as $E_{y y}$. In this plane, $\left|\operatorname{LAM}_{x}\right|^{2}$ and $\left|\mathrm{LAM}_{y}\right|^{2}$ appear weak and blurred as their corresponding gradient components $\left(E_{x y}\right.$ and $\left.E_{z y}\right)$ exhibit no spatial contrast outside the octopod. In the Methods section, we provide a detailed explanation of the relation between the field gradient components, plus further comparisons with the LAM components for a different incidence plane. The analyzed octopod produces $\gamma_{x y}=6.7$ and $\gamma_{x z}=11.9$ due to the high level of LAM localization caused by the sharp tips of the structure (Figure 5c,e).

The results shown in Figures 3-5 clearly show that the enhancement patterns for the LAM and field gradients are very similar for a broad range of geometries. This finding is a strong argument supporting the use of the much simpler LAM quantity as a figure of merit when optimizing nanostructures to provide large field gradients. We now turn our attention to quadrupolar molecular rotational transitions and show how the LAM can be physically related to the mechanical rotation of molecules.

By considering a simple rigid rotor molecular model, consisting of two homonuclear masses $m$ with bond length $a$, the energy associated with its rotational motion is ${ }^{71}$

$$
E_{\mathrm{rot}}=\frac{L^{2}}{2 I}
$$




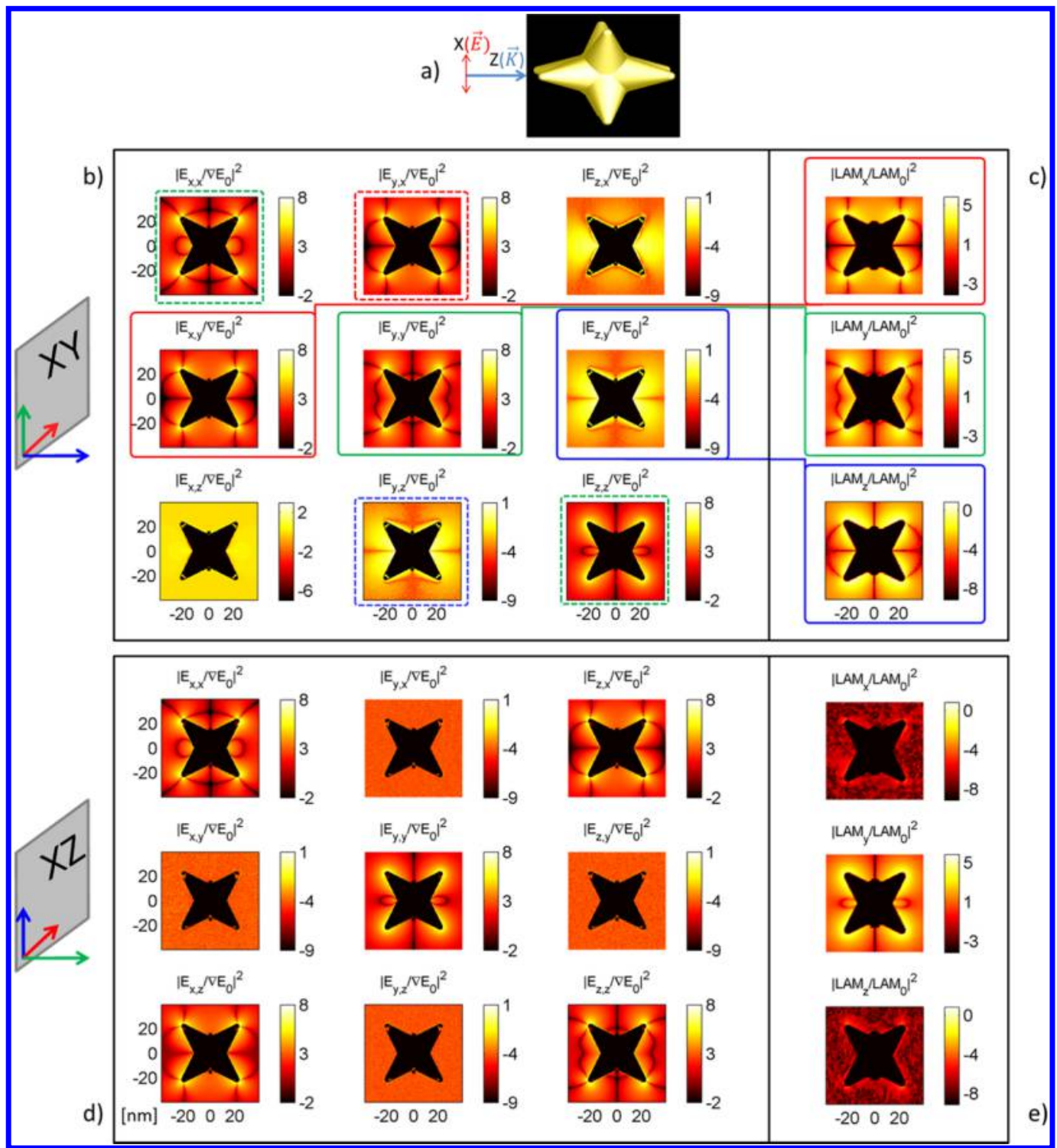

Figure 5. Illustration of the connection between normalized LAM and electric field gradients in logarithmic units. (a) Schematic illustration of the octopod irradiated by a plane wave in the long wavelength limit. Electric field gradient and normalized LAM of the octopod in the $x y$ plane $(b, c)$ and $x z$ plane $(\mathrm{d}, \mathrm{e})$. Colored solid lines highlight the direct connections between specific field gradients and normalized LAM components. Colored dashed squares indicate the components that can be derived from the same solid color ones due to symmetry arguments (see Methods for further details regarding electric field gradient symmetry properties). The logarithmic contour scale is different in some of the panels but defined immediately to the right of each panel. Vertical and horizontal axes represent distances (nm). LAM values are normalized to $\operatorname{LAM}_{0}=2 \pi\left|E_{0}\right|^{2} \varepsilon_{0} R_{S}^{2} / c \lambda$.

where $L$ is the angular momentum, $I=\mu a^{2}$ is the moment of inertia, and $\mu=m / 2$ is the reduced mass of the homonuclear diatomic molecule. Since the AM is quantized as $L^{2}=J(J+1) \hbar^{2}$, the rotational energy can be expressed as

$$
E_{\text {rot }}=\frac{\hbar^{2}}{2 I} J(J+1)
$$

The selection rule for quadrupolar transitions is $\Delta J= \pm 2$, which corresponds to an excitation energy

$$
\Delta E_{+2}=\frac{\hbar^{2}}{I}(2 J+3) \text { and } \Delta E_{-2}=\frac{\hbar^{2}}{I}(2 J-1)
$$

where $J$ is the initial rotational state. From a semiclassical point of view, the field induces a torque, mediated by the absorption of a photon, that changed the molecular AM. ${ }^{47}$ The energy increase $\Delta E_{J}$ involved in a pure rotational transition is directly related to its increase of the AM, given by

$$
\Delta L_{i f}=\hbar\left(\sqrt{J_{f}\left(J_{f}+1\right)}-\sqrt{J_{i}\left(J_{i}+1\right)}\right)
$$

Now the torque can be defined as the change of the molecular AM multiplied by the transition rate (1)

$$
\tau=\Delta L_{i f} \Gamma_{i f}
$$

For quadrupolar transitions, the relationship between torque and LAM thus becomes

$$
\begin{aligned}
\tau= & \left(\sqrt{\left(J_{i}+2\right)\left(J_{i}+3\right)}-\sqrt{J_{i}\left(J_{i}+1\right)}\right) \\
& \times \xi_{R} \frac{2 \pi}{\hbar} \overline{\mid \operatorname{LAM|}^{2}} Q^{2} \delta\left(\omega_{i f} \pm \omega\right)
\end{aligned}
$$


where $\xi_{R}$ is a coefficient capturing the conversion from gradient to LAM as defined in the Methods section and $\Gamma_{i f}=\Gamma_{i f, q u a d r u p o l e}$ was employed. We note that the double-dot operator could be dropped since the random mutual orientation between a molecule and the EM field allows for substituting quadrupole moment and gradient tensors with scalar quantities; see Methods for this LAM-dependent derivation and further details. As an example, for $\mathrm{H}_{2}$ molecules, the torque associated with the $J=0 \rightarrow 2$ transition is

$$
\tau_{\mathrm{H}_{2}}=\sqrt{6} \xi_{R} \frac{2 \pi}{\hbar} \overline{|\mathrm{LAM}|^{2}} Q^{2} \delta\left(\omega_{i f} \pm \omega\right)
$$

Thus for a given quadrupolar moment $Q$ the torque $\tau$ will be proportional to $|\mathrm{LAM}|^{2}$, showing the relevance of LAM both for transition rates and for torque. More generally, both the torque and quadrupolar transition rates will be enhanced by a factor of $(\lambda / d)^{2}$ compared to PW excitation when the molecule is near a spherical metallic NP of diameter $d$. For dipole-forbidden, purely rotational molecular transitions, the resonance wavelengths fall typically in the interval from $30 \mu \mathrm{m}\left(\right.$ for $\left.\mathrm{H}_{2}\right)$ to $4 \mathrm{~mm}$ (for $\mathrm{CO}_{2}$ ), ${ }^{72,73}$ yielding to transition rate enhancements up to $\sim 10^{7}$ and $\sim 10^{11}$, respectively, for $d=10 \mathrm{~nm} \mathrm{NPs}$. Even larger enhancements are possible for higher $n$-order transitions that exhibit $(\lambda / d)^{2 n}$ scaling, indicating that LAM optimization for metallic nanostructures can dramatically enhance weak multipolar rotational transition rates.

This analysis shows that LAM optimization could also be useful for designing metallic nanostructures that could induce rotation in classical objects. In fact, the electromagnetic AM density from which LAM is derived contains both spin (SAM) and orbital (OAM) angular momentum components. ${ }^{41,53}$ Properly designed nanostructures can scatter linearly polarized electromagnetic fields containing no OAM to exert a local torque on embedding media or attached analytes, causing a rotation of the scatterer. ${ }^{74,75}$ As an alternative to the approach presented here, a hydrodynamic heuristic that considers Poynting vector topological features could be used to quantify optical energy circulation near nanoscale metallic structures. Here, Poynting vector phase singularities are related to optical vortices, which in turn can be associated with effective "nanogear transmission". ${ }^{76-79}$ In this context, LAM can help describe and optimize these phenomena since it is directly related to the amount of AM exchanged between the field and the system. The LAM concept can also be easily extended to describe torques and interactions exerted by circularly polarized or Laguerre-Gaussian beams which carry both finite SAM and $\mathrm{OAM}^{80-83}$ and can influence the optical response of plasmonic systems. $^{84}$

\section{CONCLUSIONS}

We have introduced the concept of local angular momentum as a figure of merit for the design and optimization of nanostructures capable of providing large local field gradients. In contrast to the nine-component electric field gradient matrix, the LAM has three components directly related to the AM of the field and is based on integrated fields that are much more suitable for conventional electromagnetic simulation. We have explicitly shown how the LAM can be used as a measure of the ability of a nanostructure to enhance multipolar transitions, a feature that is particularly important for the spectroscopic detection of molecules that do not possess a permanent dipole moment. Because the LAM can be used to estimate quantum mechanical molecular transition rates and classical torque capable of rotating a nearby object, we believe that LAM will become an important figure of merit for the rational design of nanostructures for these applications.

\section{METHODS}

Derivation of Transition Rates for Multipolar Transitions. Using the interaction Hamiltonian of eq 3, we first derive an explicit expression for the dipole transition rate: ${ }^{39,85}$

$$
\begin{aligned}
& \left.\Gamma_{i f}\right|_{\text {dipole }}=\frac{2 \pi}{\hbar^{2}} \mid \int \varphi_{i}^{*} \text { er }\left.\cdot \mathbf{E}_{0} \varphi_{f} \mathrm{~d} \tau\right|^{2} \delta\left(\omega_{i f} \pm \omega\right) \\
& \quad=\frac{2 \pi}{\hbar^{2}} M_{\text {dipole }}^{2} \delta\left(\omega_{i f} \pm \omega\right)
\end{aligned}
$$

where $\hat{\epsilon}=\mathbf{E}_{0} /\left|\mathbf{E}_{0}\right|$ is a unit vector in the direction of the electric field, and $M_{\text {dipole }}$ is the transition dipole matrix element defined as

$$
M_{\text {dipole }}=\left|E_{0} \int \varphi_{i}^{*} \hat{\epsilon} \cdot \boldsymbol{\mu} \varphi_{f} \mathrm{~d} \tau\right|
$$

for initial and final wave functions $\varphi_{i}$ and $\varphi_{f}$, respectively. Note that $\Gamma_{i f}$ depends on the intensity of the interacting electric field and on the spatial distribution of the wave functions, usually expressed by spherical harmonics. Most molecules have permanent dipole moments whose strength depends on the symmetry relationship among $\varphi_{i}, \varphi_{f}$ and $\mathbf{r}$ such that the integral over the molecular volume is nonzero. Since $\mathbf{r}$ has a natural odd parity for coordinate inversion, the initial and final eigenfunctions must have different parity for a dipolar transition which, from the properties of spherical harmonics $Y_{l m}(\theta, \phi)$, are given by $(-1)^{l} .^{86}$ This and the conservation of AM produce the $\Delta l= \pm 1$ selection rule for dipole-allowed transitions. The selection rule for the projection quantum number $m$ is $\Delta m=0, \pm 1$ for linearly polarized light, and for electronic excitations, spin $S$ must be included, so conservation of total angular momentum $\mathbf{J}=\mathbf{L}+\mathbf{S}$ gives $\Delta J=0, \pm 1$. Since the overall transition rate scales as $E_{0}^{2}$, dipolar transitions are enhanced by high electric field amplitudes $E_{0}$.

Regarding the second term in eq 3, the quadrupole moment of a molecule is a traceless symmetric tensor within a Cartesian coordinate system given by a $3 \times 3$ matrix:

$$
\mathbf{Q}=\frac{1}{2} \int \rho(r)\left(\begin{array}{ccc}
3 x^{2}-r^{2} & 3 x y & 3 x z \\
3 y x & 3 y^{2}-r^{2} & 3 y z \\
3 z x & 3 z y & 3 z^{2}-r^{2}
\end{array}\right) \mathrm{d}^{3} r
$$

For a linear molecule, only the diagonal components are nonzero, the largest of which is the component along the molecular axis. ${ }^{87}$ The gradient of the electric field is also a matrix, so the quadrupolar transition rate takes the form

$$
\Gamma_{i f, \text { quadrupole }}=\frac{2 \pi}{\hbar^{2}} M_{\text {quadrupole }}^{2} \delta\left(\omega_{i f} \pm \omega\right)
$$

where $M_{\text {quadrupole }}$ is defined as

$$
M_{\text {quadrupole }}=|-|(\nabla \mathbf{E})_{0}\left|\int \varphi_{i}^{*} \hat{\eta}: \mathbf{Q} \varphi_{f} \mathrm{~d} \tau\right|
$$

and $\hat{\eta}=(\nabla \mathbf{E})_{0} / I(\nabla \mathbf{E})_{0} \mid$, where $\left|(\nabla \mathbf{E})_{0}\right|=\sqrt{\sum_{i j}\left|\left(E_{i j}\right)_{0}\right|^{2}}$ and $E_{i j}=\frac{\partial E_{i}}{\partial j} ; \hat{\eta}$ is a $3 \times 3$ matrix that captures the relationship between gradient and quadrupole moment components. The quadrupolar selection rules differ from the dipolar selection rules because $Q$ has an even parity: $\Delta l=0, \pm 2$ and $\Delta J=0, \pm 1, \pm 2$ (with exceptions: $\left.0 \leftrightarrow 0, \frac{1}{2} \leftrightarrow \frac{1}{2}, 0 \leftrightarrow 1\right)$. Selection rules with $\Delta J>1$ require a contribution from OAM in addition to the SAM from the photon in order to conserve AM.

Using eq 19 to calculate the quadrupole-mediated transition rate involves the contraction of the quadrupole and gradient tensors. However, the first nonzero term of a multipolar expansion is generally 
origin independent, ${ }^{87}$ so we can choose it to specify the multipole moment tensor. Thus, for a linear molecule oriented parallel to the $z$-axis, we can choose the dominant term as

$$
\mathbf{Q} \approx Q=Q_{z z}=-2 Q_{x x}=-2 Q_{y y}
$$

Moreover, the orientation of the molecule with respect to the EM field is usually random, so only the average of the nine gradient components enters the transition rate, and we can recast the quadrupolar transition rate into a more compact form:

$$
\left\{\begin{array}{c}
\Gamma_{i f, \text { quadrupole }}=\frac{2 \pi}{\hbar^{2}} \overline{\left|(\nabla \mathbf{E})_{0}\right|^{2}} Q^{2} \delta\left(\omega_{i f} \pm \omega\right) \\
\overline{\left|(\nabla \mathbf{E})_{0}\right|^{2}}=\frac{1}{9} \sum_{\alpha, b=1}^{3}\left|\left(\nabla E_{\alpha \beta}\right)_{0}\right|^{2}
\end{array}\right.
$$

This expression allows us to relate the quadrupolar transition rates to the LAM as

$$
\left\{\begin{array}{c}
\Gamma_{i f, \text { quadrupole }}=\xi_{R} \frac{2 \pi}{\hbar^{2}} \overline{|\mathrm{LAM}|^{2}} Q^{2} \delta\left(\omega_{i f} \pm \omega\right) \\
\overline{|\mathrm{LAM}|^{2}}=\frac{1}{3} \sum_{\alpha=1}^{3}\left|\left(\operatorname{LAM}_{\alpha}\right)\right|^{2}
\end{array}\right.
$$

where $\xi_{R}$ is a coefficient capturing the conversion from gradient to LAM. For small values of $R$ so that $\nabla E$ is approximately constant within the integration volume $V$, we have $\xi_{R}=\left(\frac{1}{3} \frac{1}{c^{2} Z} R^{2} E_{0}\right)^{-2}$, where $c$ is the velocity of light, $Z=\sqrt{\frac{\mu_{0}}{\varepsilon_{0}}}$ is the vacuum impedance, $R$ is the integration radius, and $E_{0}$ is the amplitude of the incident electric field. If the vacuum ambient is replaced with a dielectric medium, $c$ and $Z$ will be altered by its permittivity.

Derivation of Equations 5-7. For transitions mediated by higher-order moments, we calculate how the field gradient is enhanced near a NP compared to a PW. We start by considering the spatial $(\mathbf{r})$ and temporal $(t)$ dependence of the PW electric field

$$
\mathbf{E}_{\mathrm{PW}}(\mathbf{r}, t)=\mathbf{E}_{0} e^{-i \mathbf{k} \cdot \mathbf{r}} e^{i \omega t}
$$

where $k=\frac{2 \pi}{\lambda} \hat{k}$ is the wave vector, and $\omega$ is the wave angular frequency. Successively differentiating eq 23 , it is easy to see that the modulus of the $n$th spatial derivative is

$$
\left|\mathbf{E}^{(n)}(\mathbf{r}, t)\right|_{\mathrm{PW}}=k^{n} E_{0}
$$

A metallic NP interacting with an electromagnetic wave whose wavelength is much larger than the LSPR $\left(\lambda \gg \lambda_{\text {LSPR }}\right)$ displays a dipolar response with a field described by ${ }^{41}$

$$
\begin{aligned}
\mathbf{E}_{\mathrm{NP}}(\mathbf{r}, t)= & \frac{1}{4 \pi \varepsilon_{0}}\left\{k^{2}(\mathbf{n} \times \mathbf{p}) \times \mathbf{n} \frac{e^{i k r}}{r}+[3 \mathbf{n}(\mathbf{n} \cdot \mathbf{p})-\mathbf{p}]\right. \\
& \left.\times\left(\frac{1}{r^{3}}-\frac{i k}{r^{2}}\right) e^{i k r}\right\} e^{i \omega t}
\end{aligned}
$$

Here, $\mathbf{n}$ is a unit vector pointing along $r$, and $\mathbf{p}=\int \mathbf{r} \rho(\mathbf{r}) \mathrm{d}^{3} r$ is the dipole moment associated with the oscillating charges within the NP. For regions very close to the NP, $k r \rightarrow 0$ and the $r^{-3}$ term dominates, so only the electrostatic part remains. Since $\mathbf{n} \cdot \mathbf{p}=0$ for radiation perpendicular to the dipole, eq 25 simplifies to

$$
\mid \mathbf{E}_{\mathrm{NP}}(\mathbf{r}, t) \|_{\mathbf{n} \cdot \mathbf{p}=0, k r \rightarrow 0} \approx-\frac{1}{4 \pi \varepsilon_{0}} \frac{p}{r^{3}} e^{i \omega t}
$$

If the NP has diameter $d$, then $p \propto \sigma d^{3}$, where $\sigma$ is the surface charge density induced on the NP surface. It follows that $\left|\nabla_{r} E_{\mathrm{NP}}\right| \approx \frac{3}{4 \pi \varepsilon_{0}} \frac{\sigma d^{3}}{r^{4}}$, and for regions close to the surface $(r \rightarrow d)$, we obtain $\left|\nabla_{r} E_{\mathrm{NP}}\right| \approx \frac{3}{4 \pi \varepsilon_{0}} \frac{\sigma}{d}$. In general, the $n$th derivative behaves as

$$
\left|\mathbf{E}^{(n)}(\mathbf{r}, t)\right|_{\mathrm{NP}} \sim \frac{\sigma}{\varepsilon_{0}} \frac{1}{d^{n}}
$$

Now $\sigma / \varepsilon_{0}$, expressed in $\mathrm{V} / \mathrm{m}$, corresponds to the electric field induced on the NP by the external radiation, and dividing this by the amplitude of the incident radiation $E_{0}$ gives the electric field enhancement $\epsilon \sim \frac{1}{\varepsilon_{0}} \frac{\sigma}{E_{0}}$. The gradient enhancement factor for the $n$ th-order term may then be expressed in terms of the electric field enhancement $\epsilon$ as

$$
\eta_{n}=\frac{\Gamma_{n, \mathrm{NP}}}{\Gamma_{n, \mathrm{PW}}}=\left(\frac{\left|\mathbf{E}^{(n)}(\mathbf{r}, t)\right|_{\mathrm{PW}}}{\left|\mathbf{E}^{(n)}(\mathbf{r}, t)\right|_{\mathrm{NP}}}\right)^{2} \sim\left(\epsilon \frac{1}{d^{n} k^{n}}\right)^{2} \approx \epsilon^{2}\left(\frac{\lambda}{d}\right)^{2 n}
$$

Note that even at wavelengths far from the LSPR, where $\epsilon \sim 1$, higherorder transitions can be significantly enhanced for small NPs with $d \ll \lambda$.

Comparison between FDTD and FEM Calculation. In order to test the accuracy of the numerical approach, Figure 6 compares the LAM

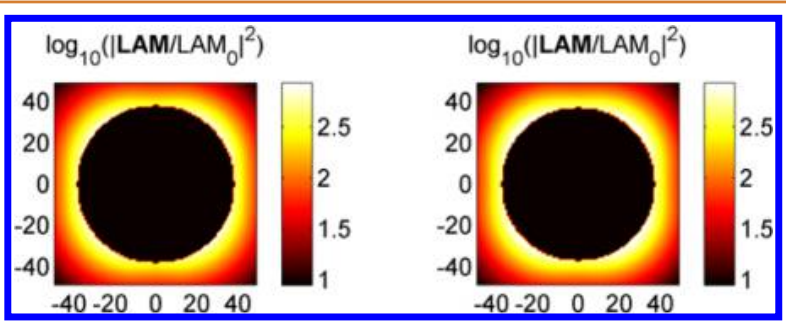

Figure 6. Comparison between Mie theory (left) and FDTD calculation (right) of normalized LAM for a $d=70 \mathrm{~nm}$ gold sphere. A logarithmic scale is used to illustrate the excellent agreement. The grid size used in the FDTD simulations is $0.25 \mathrm{~nm}$, with $R_{\mathrm{S}}=1 \mathrm{~nm}$. LAM values are normalized to $\mathrm{LAM}_{0}=2 \pi\left|E_{0}\right|^{2} \varepsilon_{0} R_{\mathrm{S}}{ }^{2} / c \lambda$.

calculated by Mie theory (left) and by FDTD (right) for a gold sphere of $d=70 \mathrm{~nm}$ and an FDTD grid size of $0.25 \mathrm{~nm}$. Clearly, the approaches produce almost identical results, confirming that the FDTD method may be reliably used to calculate LAM for more complex geometries.

In Figure 7, FDTD and FEM results are compared in the case of a conical geometry with different tip radii $(2,3.5$, and $5 \mathrm{~nm})$. The two

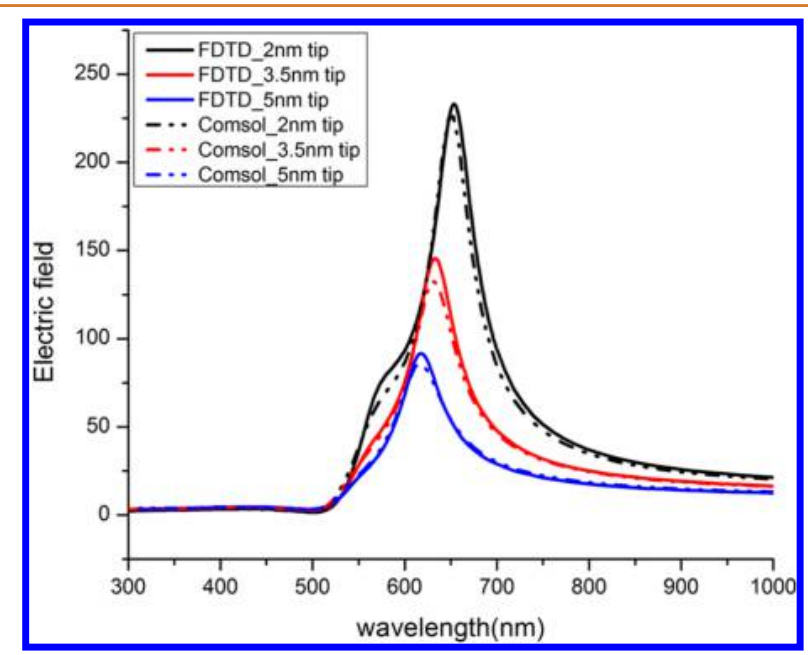

Figure 7. Enhancement spectra of the electric near-field, calculated $1 \mathrm{~nm}$ above conical structures $(70 \mathrm{~nm}$ wide and $100 \mathrm{~nm}$ high) with different tip radii $(2,3.5$, and $5 \mathrm{~nm})$. Solid lines: FDTD calculations. Dashed lines: FEM calculations.

methods clearly agree in each of the calculated cases for field enhancements evaluated $1 \mathrm{~nm}$ from the tip. 
Effect of the Integration Radius and Surface Discretization on LAM Calculation. For simplicity, within this section only, LAM values will not be divided by normalization factor, $\mathrm{LAM}_{0}$. An important factor in the calculation of LAM is the integration volume. It is obvious that different integration volumes will return different LAM values. For the long wavelength case, or any time the quasistatic approximation can be applied, Figure 8a reveals a nonlinear quartic

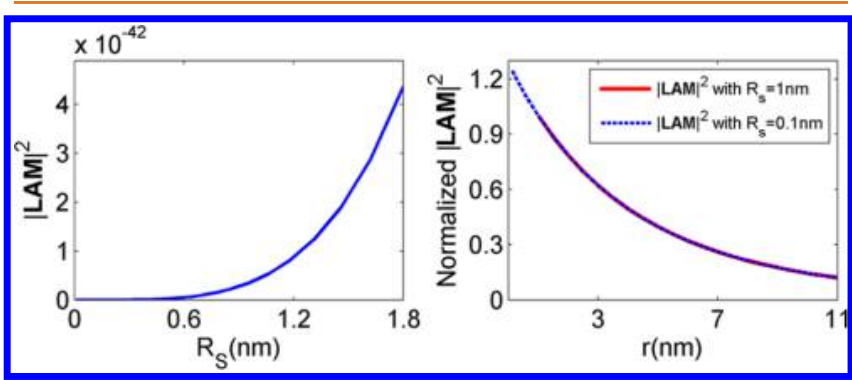

Figure 8. (a) $|L A M|^{2}$ in the long wavelength regime, showing that it is proportional to $R_{\mathrm{S}}{ }^{4}$. (b) Normalized $|\mathrm{LAM}|^{2}$ calculated with different integration radii $R_{S}(0.1$ and $1 \mathrm{~nm})$ at different distances from the sphere surface for a $d=70 \mathrm{~nm}$ nanosphere.

relation between the integration radius $R_{\mathrm{S}}$ (of volume $V$ ) and $|\mathrm{LAM}|^{2}$, indicating that LAM is proportional to $R_{S}^{2}$. This quadratic dependence can be understood from eq 9, where LAM has been decomposed in term of the gradient components with quadratic $r_{x}^{2}, r_{y}^{2}, r_{z}^{2}$ terms that, when integrated over spherical coordinates (eq 8), give the $R_{S}{ }^{4}$ relationship of $|\mathrm{LAM}|^{2}$.

This relationship is important if we consider another issue that concerns the LAM integration. At positions so close to the NP that the integration radius $R_{\mathrm{S}}$ is larger than the distance $u$ from the surface, inaccurate LAM values are produced when the integration volume intersects the NP surface. Since the mesh size cannot be infinitely small for FDTD simulations, LAM cannot be calculated immediately adjacent to the NP. However, when $|\mathrm{LAM}|^{2}$ is normalized by its maximum value for a given integration radius (Figure $8 b$ ), we see LAM follows a predictable, monotonic trend for decreasing integration radii. Thus, LAM calculations performed with relatively large $R_{\mathrm{S}}$ can be used to extrapolate LAM values close to the NP where $R_{\mathrm{S}}>u$.

Derivation of Equation 9. The connection between LAM and gradient $\nabla \mathbf{E}$ is best observed by explicitly expressing the LAM components in terms of the components of the electric field gradient

$$
\begin{aligned}
\left(\begin{array}{c}
\operatorname{LAM}_{x} \\
\operatorname{LAM}_{y} \\
\operatorname{LAM}_{z}
\end{array}\right)= & \frac{\varepsilon \mu}{V} \sum\left(\begin{array}{c}
r_{y}\left(E_{x} H_{y}-E_{y} H_{x}\right)-r_{z}\left(E_{z} H_{x}-E_{x} H_{z}\right) \\
r_{z}\left(E_{y} H_{z}-E_{z} H_{y}\right)-r_{x}\left(E_{x} H_{y}-E_{y} H_{x}\right) \\
r_{x}\left(E_{z} H_{x}-E_{x} H_{z}\right)-r_{y}\left(E_{y} H_{z}-E_{z} H_{y}\right)
\end{array}\right) \\
& \sim \frac{\varepsilon \mu}{V} \sum\left(\begin{array}{c}
r_{y}^{2} E_{x y} \\
-r_{z}^{2} E_{z z}-r_{x}{ }^{2} E_{x x} \\
r_{y}{ }^{2} E_{z y}
\end{array}\right) H_{y}
\end{aligned}
$$

where $E_{i j}=\partial E_{i} / \partial j$. Considering an $x$-polarized PW traveling in the $z$ direction in the long wavelength limit, $E_{x}, E_{y} \gg E_{z}, H_{y}$ is almost a constant, and $H_{y} \gg H_{x}, H_{z}$. As long as a term that contains $H_{y}$ also includes a large $E_{i j}$, it will be much larger than any term that does not contain $H_{y}$. Moreover, we observe that for any small NP in quasistatic approximation Laplace equation $\nabla^{2} \Phi=0$ holds with $\mathbf{E}=-\nabla \Phi^{88}$ and thus $E_{x x}+E_{y y}+E_{z z}=0$ with $E_{j i}=E_{i j}$. We therefore observe, from eq 29, that LAM directly contains seven of the nine components belonging to the electric field gradient matrix which have a correspondingly dominant LAM component, and the two quantities will exhibit similar spatial distributions. The only exceptions are for $E_{x z}$ and $E_{z x}$, which share the same pattern. In other words, for any point with a large LAM near a NP, at least one of the seven components of the electric field gradient will be large in the same region.
Take, for example, the octopod in the $x y$ plane and notice that $E_{x y}$ and $\left(E_{z z}+E_{x x}\right)$ are much larger than $E_{z y}$. We see that $\operatorname{LAM}_{x}$ and $\mathrm{LAM}_{y}$ are much larger than $\mathrm{LAM}_{z}$, and their intensity distribution patterns are similar. Equation 29 holds as long as the susceptibility tensor (i.e., the NP polarizability) is diagonal and $\mu \sim \mu_{0}$, in which case

$$
\mathbf{P}=N \alpha \mathbf{E}_{0}=\varepsilon_{0} \chi \mathbf{E}_{0}=\varepsilon_{0}\left(\begin{array}{ccc}
\chi_{x x} & 0 & 0 \\
0 & \chi_{y y} & 0 \\
0 & 0 & \chi_{z z}
\end{array}\right) \mathbf{E}_{0}
$$

This means that the optical response of the NP does not twist the incident EM field; that is, the scattering sources within the NP are oriented along the polarization of the impinging radiation. Therefore, the total incident plus scattered field preserves the same relations among the components described above.

LAM and Gradient Discrepancy for a Conical Nanostructure. To demonstrate the marginal role played by the gradient bright ring at the base of a conical nanostructure (Figure 4), we compare the gradient strength over a sphere centered at the tip (where both gradient and LAM hot spots are present) to the gradient strength over spheres centered at the base rim of the cone (where only gradient bright spots are present). In Figure 9, the signal from the tip is compared

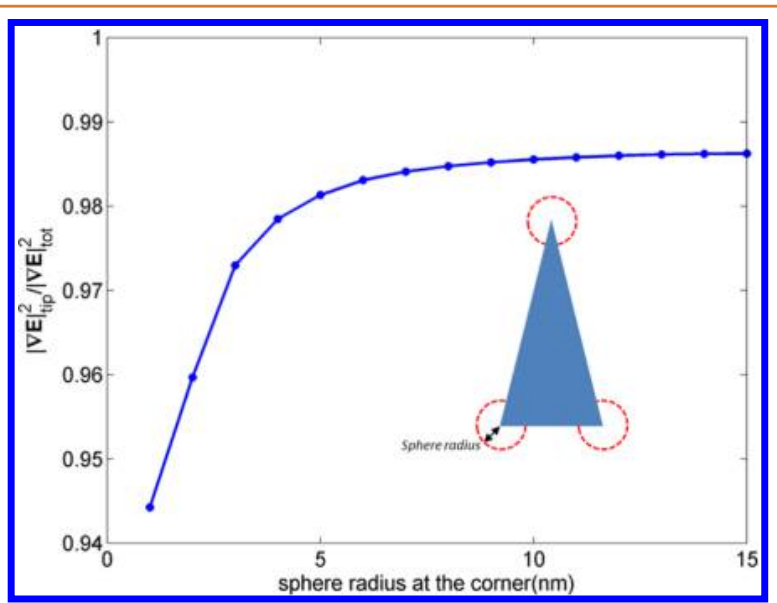

Figure 9. Fraction of the total gradient strength contributed by the tip of the conical nanostructure, as discussed in the main text. The signal is integrated over spheres whose radii change from 1 to $15 \mathrm{~nm}$.

with the total (tip + base) as the integration sphere radii change from 1 to $15 \mathrm{~nm}$.

From the plot it is clear that the dominant gradient signal comes from the tip region. Even when the integration radius is $1 \mathrm{~nm}$, so only the field gradient proximal to the surfaces contributes, more than $94 \%$ of the gradient intensity comes from the region close to the sharp tip of the cone.

Comparison of LAM and Gradient for an Octopod on a Different Incidence Plane. Figure 10 shows the LAM and gradient components in the long wavelength limit for a plane wave interacting with an octopod rotated $45^{\circ}$ in $y$ and $z$. The specific connections between LAM and gradient components can be recovered in the same manner described for Figure 5. Moreover, even if the $E_{x z}$ and $E_{z x}$ components are large, LAM still is a good figure of merit because the intensity of the diagonal components also exhibits large values.

Rigid Rotor Molecular Model. In order to obtain a more intuitive understanding of the relationship between electric field gradients and $\mathrm{AM}$, we now consider a simple rigid rotor molecular model, consisting of two homonuclear masses $m$ with bond length $a$ interacting with a force field $F$. This type of molecule possesses no permanent dipole moment, and half of the quantized rotational states are absent because of symmetry. However, it has a nonzero quadrupole moment which may be calculated, ${ }^{89-92}$ and a quadrupolar transition $J \rightarrow J+2$ may occur if the molecule senses a sufficiently strong field gradient $\left(\mathbf{Q}:(\nabla \mathbf{E})_{0}\right.$ 


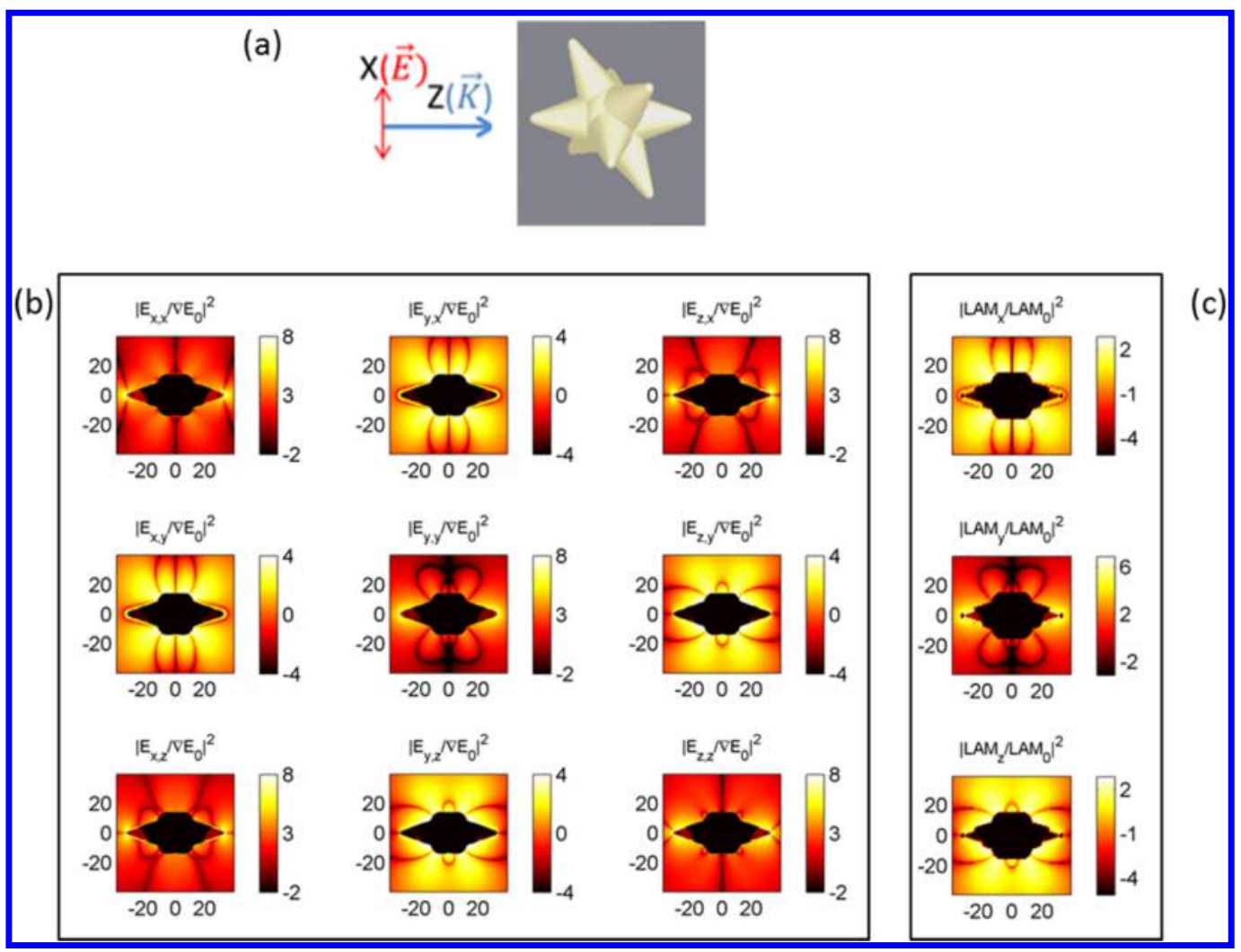

Figure 10. (a) Sketch of the octopod rotated $45^{\circ}$ in $y$ and $z$. (b) Gradient and (c) normalized LAM components in the $X Z$ plane for octopod-shaped nanostructures. Logarithmic units. Note $E_{x z}, E_{z x}$, and diagonal components are each large. LAM values are normalized to $\operatorname{LAM}_{0}=2 \pi\left|E_{0}\right|^{2} \varepsilon_{0} R_{\mathrm{S}}^{2} / c \lambda$.

from eq 3), conservation of energy $(\Delta E=h \nu)$, and conservation of AM $(\Delta J=2)$. Indeed, the quadrupole moment is the measure of how sensitive the molecule is to a field gradient, and LAM is a measure of the AM available in the local field. From our now established connection between field gradient and LAM, we expect that molecules located in regions of large LAM will experience the greatest transition rate enhancements.

To illustrate this, consider that when $F$ is constant along the symmetry axis, its gradient is zero, the molecule does not experience a torque, and the field does not induce a rotation (Figure 11a). If an external field with

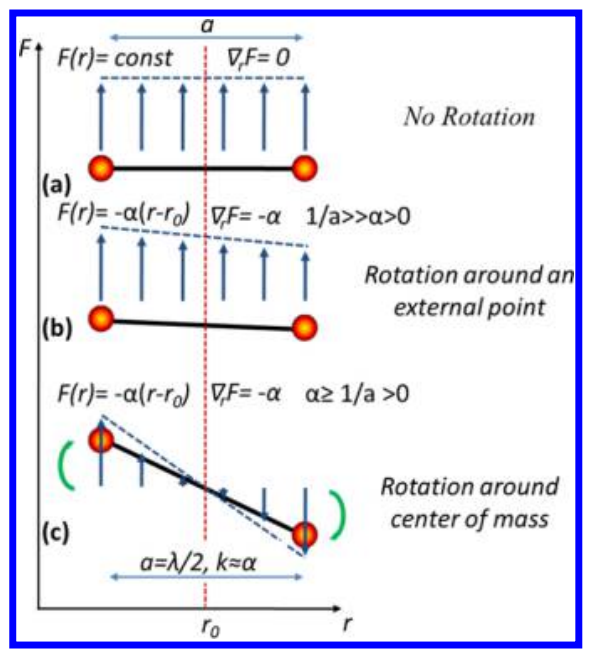

Figure 11. (a) Constant force field $F$ acts on a rigid rotor, causing no rotation. (b) Gradually varying field $F$ interacts with the rotor, inducing a rotation with respect to an external point. (c) Steeply varying field $F$ induces a rotation around an internal point. an imposed orbital angular momentum is used, $F$ decreases linearly with steepness $-\alpha$ in the $r$ direction such that $(1 / a) \gg \alpha>0$. In this case, the molecule may be induced to orbit a distant, external point, as described in Figure $11 \mathrm{~b}$, but again no rotational transition occurs. Finally, if the gradient is steep and the force decreases so rapidly that its direction changes across the molecule (i.e., $\alpha \gg(1 / a)>0$ ), then a rotational transition may be induced (see Figure 11c). If $F$ is the oscillating field of an electromagnetic PW, then the molecule can "sense" the gradient and start rotating only when the wave vector $k \gtrsim 1 / a($ i.e., $\lambda \lesssim a)$.

\section{AUTHOR INFORMATION}

\section{Corresponding Authors}

*E-mail: alessandro.alabastri@rice.edu.

*E-mail: everitt@phy.duke.edu.

*E-mail: nordland@rice.edu.

\section{Author Contributions}

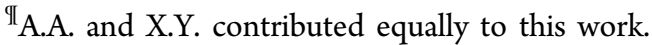

\section{Notes}

The authors declare no competing financial interest.

\section{ACKNOWLEDGMENTS}

The authors acknowledge the early support of this project by M. Goodman, and financial support by grants from DARPA and the Robert A. Welch Foundation (Grant No. C-1222). A.M. acknowledges financial support from the Department of Physics and Astronomy and the College of Arts and Sciences of the University of New Mexico.

\section{REFERENCES}

(1) De Angelis, F.; Gentile, F.; Mecarini, F.; Das, G.; Moretti, M.; Candeloro, P.; Coluccio, M. L.; Cojoc, G.; Accardo, A.; Liberale, C.; Zaccaria, R. P.; Perozziello, G.; Tirinato, L.; Toma, A.; Cuda, G.; 
Cingolani, $R_{. i}$ Di Fabrizio, E. Breaking the Diffusion Limit with SuperHydrophobic Delivery of Molecules to Plasmonic Nanofocusing Sers Structures. Nat. Photonics 2011, 5, 682-687.

(2) Crozier, K. B.; Zhu, W.; Wang, D.; Lin, S.; Best, M. D.; Camden, J. P. Plasmonics for Surface Enhanced Raman Scattering: Nanoantennas for Single Molecules. IEEE J. Sel. Top. Quantum Electron. 2014, 20, 152-162.

(3) Etchegoin, P. G.; Le Ru, E. C. A Perspective on Single Molecule Sers: Current Status and Future Challenges. Phys. Chem. Chem. Phys. 2008, 10, 6079-6089.

(4) Fromm, D. P.; Sundaramurthy, A.; Kinkhabwala, A.; Schuck, P. J.; Kino, G. S.; Moerner, W. E. Exploring the Chemical Enhancement for Surface-Enhanced Raman Scattering with Au Bowtie Nanoantennas. J. Chem. Phys. 2006, 124, 61101.

(5) Nie, S.; Emory, S. R. Probing Single Molecules and Single Nanoparticles by Surface-Enhanced Raman Scattering. Science (Washington, DC, U. S.) 1997, 275, 1102-1106.

(6) Xu, J. J.; Zhang, L.; Gong, H.; Homola, J.; Yu, Q. M. Tailoring Plasmonic Nanostructures for Optimal SERS Sensing of Small Molecules and Large Microorganisms. Small 2011, 7, 371-376.

(7) Das, G.; Chirumamilla, M.; Toma, A.; Gopalakrishnan, A.; Zaccaria, R. P.; Alabastri, A.; Leoncini, M.; Di Fabrizio, E. Plasmon Based Biosensor for Distinguishing Different Peptides Mutation States. Sci. Rep. 2013, 3, 1792.

(8) Petryayeva, E.; Krull, U. J. Localized Surface Plasmon Resonance: Nanostructures, Bioassays and Biosensing-a Review. Anal. Chim. Acta 2011, 706, 8-24.

(9) Zhang, S.; Bao, K.; Halas, N. J.; Xu, H.; Nordlander, P. SubstrateInduced Fano Resonances of a Plasmonic Nanocube: A Route to Increased-Sensitivity Localized Surface Plasmon Resonance Sensors Revealed. Nano Lett. 2011, 11, 1657-1663.

(10) Mubeen, S.; Lee, J.; Lee, W.-r.; Singh, N.; Stucky, G. D.; Moskovits, M. On the Plasmonic Photovoltaic. ACS Nano 2014, 8, 6066-6073.

(11) Atwater, H. A.; Polman, A. Plasmonics for Improved Photovoltaic Devices. Nat. Mater. 2010, 9, 205-213.

(12) Bai, W.; Gan, Q.; Bartoli, F.; Zhang, J.; Cai, L.; Huang, Y.; Song, G. Design of Plasmonic Back Structures for Efficiency Enhancement of Thin-Film Amorphous Si Solar Cells. Opt. Lett. 2009, 34, 3725-3727.

(13) Ilic, O.; Jablan, M.; Joannopoulos, J. D.; Celanovic, I.; Soljacic, M. Overcoming the Black Body Limit in Plasmonic and Graphene near-Field Thermophotovoltaic Systems. Opt. Express 2012, 20, A366-84.

(14) Polman, A.; Atwater, H. A. Photonic Design Principles for Ultrahigh-Efficiency Photovoltaics. Nat. Mater. 2012, 11, 174-177.

(15) Adleman, J. R.; Boyd, D. A.; Goodwin, D. G.; Psaltis, D. Heterogenous Catalysis Mediated by Plasmon Heating. Nano Lett. 2009, 9, 4417-4423.

(16) Hou, W.; Cronin, S. B. A Review of Surface Plasmon Resonance-Enhanced Photocatalysis. Adv. Funct. Mater. 2013, 23, $1612-1619$.

(17) Kubus, L.; Erdogan, H.; Cetin, S. S.; Biskin, E.; Demirel, G. Plasmon-Enhanced Photocatalysis on Anisotropic Gold Nanorod Arrays. ChemCatChem 2013, 5, 2973.

(18) Honda, M.; Kumamoto, Y.; Taguchi, A.; Saito, Y.; Kawata, S. Plasmon-Enhanced UV Photocatalysis. Appl. Phys. Lett. 2014, 104, 061108.

(19) Mukherjee, S.; Libisch, F.; Large, N.; Neumann, O.; Brown, L. V.; Cheng, J.; Lassiter, J. B.; Carter, E. A.; Nordlander, P.; Halas, N. J. Hot Electrons Do the Impossible: Plasmon-Induced Dissociation of $\mathrm{H}_{2}$ on Au. Nano Lett. 2013, 13, 240-247.

(20) Tam, F.; Goodrich, G. P.; Johnson, B. R.; Halas, N. J. Plasmonic Enhancement of Molecular Fluorescence. Nano Lett. 2007, 7, 496501.

(21) Baffou, G.; Kreuzer, M. P.; Kulzer, F.; Quidant, R. Temperature Mapping near Plasmonic Nanostructures Using Fluorescence Polarization Anisotropy. Opt. Express 2009, 17, 3291-3298.

(22) Bharadwaj, P.; Beams, R.; Novotny, L. Nanoscale Spectroscopy with Optical Antennas. Chem. Sci. 2011, 2, 136-140.
(23) Fu, Y.; Zhang, J.; Lakowicz, J. R. Largely Enhanced SingleMolecule Fluorescence in Plasmonic Nanogaps Formed by Hybrid Silver Nanostructures. Langmuir 2013, 29, 2731-2738.

(24) Khatua, S.; Paulo, P. M. R.; Yuan, H.; Gupta, A.; Zijlstra, P.; Orrit, M. Resonant Plasmonic Enhancement of Single-Molecule Fluorescence by Individual Gold Nanorods. ACS Nano 2014, 8, 4440-4449.

(25) Hao, Q.; Du, D.; Wang, C.; Li, W.; Huang, H.; Li, J.; Qiu, T.; Chu, P. K. Plasmon-Induced Broadband Fluorescence Enhancement on Al-Ag Bimetallic Substrates. Sci. Rep. 2014, 4, 6014.

(26) Tong, L.; Wei, Q.; Wei, A.; Cheng, J.-X. Gold Nanorods as Contrast Agents for Biological Imaging: Optical Properties, Surface Conjugation, and Photothermal Effects. Photochem. Photobiol. 2009, $85,21$.

(27) Homola, J. Surface Plasmon Resonance Sensors for Detection of Chemical and Biological Species. Chem. Rev. (Washington, DC, U. S.) 2008, 108, 462-493.

(28) Giugni, A.; Torre, B.; Toma, A.; Francardi, M.; Malerba, M.; Alabastri, A.; Zaccaria, R. P.; Stockman, M. I.; Di Fabrizio, E. HotElectron Nanoscopy Using Adiabatic Compression of Surface Plasmons. Nat. Nanotechnol. 2013, 8, 845-852.

(29) Lindquist, N. C.; Nagpal, P.; McPeak, K. M.; Norris, D. J.; Oh, S.-H. Engineering Metallic Nanostructures for Plasmonics and Nanophotonics. Rep. Prog. Phys. 2012, 75, 036501.

(30) Zhang, J.; Zhang, L.; Xu, W. Surface Plasmon Polaritons: Physics and Applications. J. Phys. D: Appl. Phys. 2012, 45, 113001.

(31) Moskovits, M.; DiLella, D. P. Intense Quadrupole Transitions in the Spectra of Molecules near Metal Surfaces. J. Chem. Phys. 1982, 77, $1655-1660$.

(32) Filter, R.; Mühlig, S.; Eichelkraut, T.; Rockstuhl, C.; Lederer, F. Controlling the Dynamics of Quantum Mechanical Systems Sustaining Dipole-Forbidden Transitions Via Optical Nanoantennas. Phys. Rev. B: Condens. Matter Mater. Phys. 2012, 86, 035404.

(33) Kern, A. M.; Martin, O. J. F. Strong Enhancement of Forbidden Atomic Transitions Using Plasmonic Nanostructures. Phys. Rev. A: At., Mol., Opt. Phys. 2012, 85, 022501.

(34) Sun, M.; Zhang, Z.; Chen, L.; Sheng, S.; Xu, H. Plasmonic Gradient Effects on High Vacuum Tip-Enhanced Raman Spectroscopy. Adv. Opt. Mater. 2014, 2, 74-80.

(35) Heeres, R. W.; Zwiller, V. Subwavelength Focusing of Light with Orbital Angular Momentum. Nano Lett. 2014, 14, 4598-4601.

(36) Fang, Y.; Zhang, Z.; Chen, L.; Sun, M. Near Field Plasmonic Gradient Effects on High Vacuum Tip-Enhanced Raman Spectroscopy. Phvs. Chem. Chem. Phvs. 2015, 17, 783-794.

(37) Yannopapas, V.; Paspalakis, E. Giant Enhancement of DipoleForbidden Transitions Via Lattices of Plasmonic Nanoparticles. J. Mod. Opt. 2015, 62, 1435-1441.

(38) Meng, L.; Yang, Z.; Chen, J.; Sun, M. Effect of Electric Field Gradient on Sub-Nanometer Spatial Resolution of Tip-Enhanced Raman Spectroscopy. Sci. Rep. 2015, 5, 9240.

(39) Cohen-Tannoudji, C.; Dupont-Roc, J.; Gryndberg, G. AtomPhoton Interactions; Wiley-VCH: Weinheim, Germany, 1998.

(40) Barron, L. D.; Gray, C. G. The Multipole Interaction Hamiltonian for Time Dependent Fields. J. Phys. A: Math., Nucl. Gen. 1973, 6, 59.

(41) Jackson, J. D. Classical Electrodynamics, 3rd ed.; John Wiley \& Sons: New York, 1999.

(42) Li, W.; Pohl, T.; Rost, J. M.; Rittenhouse, S. T.; Sadeghpour, H. R.; Nipper, J.; Butscher, B.; Balewski, J. B.; Bendkowsky, V.; Löw, R; Pfau, T. A Homonuclear Molecule with a Permanent Electric Dipole Moment. Science (Washington, DC, U. S.) 2011, 334, 1110-1114.

(43) Camy-Peyret, C.; Flaud, J.-M.; Delbouille, L.; Roland, G.; Brault, J. W.; Testerman, L. Quadrupole Transitions of the $1 \leftarrow 0$ Band of $\mathrm{N}_{2}$ Observed in a High Resolution Atmospheric Spectrum. J. Phys., Lett. 1981, 42, 279-283.

(44) Tipping, R. H.; Brown, A.; Ma, Q.; Hartmann, J. M.; Boulet, C.; Liévin, J. Collision-Induced Absorption in the $\mathrm{N}_{2}$ Fundamental Band of $\mathrm{CH}_{4}$. I. Determination of the Quadrupole Transition Moment. J. Chem. Phys. 2001, 115, 8852-8857. 
(45) Hu, S. M.; Pan, H.; Cheng, C. F.; Sun, Y. R.; Li, X. F.; Wang, J.; Campargue, A.; Liu, A. W. The $\mathrm{V}=3 \leftarrow 0 \mathrm{~S}(0)$-S(3) Electric Quadrupole Transitions of $\mathrm{H}_{2}$ near $0.8 \mu \mathrm{m}$. Astrophys. I. 2012, 749, 76.

(46) Cheng, C. F.; Sun, Y. R.; Pan, H.; Wang, J.; Liu, A. W.; Campargue, A.; Hu, S. M. Electric-Quadrupole Transition of $\mathrm{H}_{2}$ Determined to $10^{-9}$ Precision. Phys. Rev. A: At., Mol., Opt. Phys. 2012, 85, 024501.

(47) Berestetskii, V. B.; Lifshitz, E. M.; Pitaevskii, L. P. Quantum Electrodynamics; Elsevier Ltd.: Oxford, UK, 1982.

(48) Bahder, T. B. Interaction of Diatomic Molecules with Photon Angular Momentum. arXiv:1212.6417 2012; http://arxiv.org/abs/ 1212.6417 (accessed Sep 30, 2015).

(49) Barnett, S. M. Optical Angular-Momentum Flux. J. Opt. B: Quantum Semiclassical Opt. 2002, 4, S7.

(50) Shevchenko, A. Electromagnetic Angular Momentum Flux Tensor in a Medium. Eur. Phys. J. D 2012, 66, 1-12.

(51) Beth, R. A. Mechanical Detection and Measurement of the Angular Momentum of Light. Phys. Rev. 1936, 50, 115-125.

(52) Simmons, J. W.; Guttmann, M. J. States Waves \& Photons: A Modern Introduction to Light; Addison-Wesley Publishing Company: Reading, MA, 1970.

(53) Stewart, A. M. Angular Momentum of the Electromagnetic Field: The Plane Wave Paradox Resolved. Eur. J. Phys. 2005, 26, 635.

(54) Stewart, A. M. Angular Momentum of Light. J. Mod. Opt. 2005, 52, 1145-1154.

(55) Bliokh, K. Y.; Rodriguez-Fortuno, F. J.; Nori, F.; Zayats, A. V. Spin-Orbit Interactions of Light. Nat. Photonics 2015, 9, 796-808.

(56) Molina-Terriza, G.; Torres, J. P.; Torner, L. Twisted Photons. Nat. Phys. 2007, 3, 305-310.

(57) O'Neil, A. T.; MacVicar, I.; Allen, L.; Padgett, M. J. Intrinsic and Extrinsic Nature of the Orbital Angular Momentum of a Light Beam. Phvs. Rev. Lett. 2002, 88, 053601.

(58) Enk, S. J. v.; Nienhuis, G. Spin and Orbital Angular Momentum of Photons. Europhys. Lett. 1994, 25, 497.

(59) Karimi, E.; Schulz, S. A.; De Leon, I.; Qassim, H.; Upham, J.; Boyd, R. W. Generating Optical Orbital Angular Momentum at Visible Wavelengths Using a Plasmonic Metasurface. Light: Sci. Appl. 2014, 3, e167.

(60) Zambrini, R.; Barnett, S. M. Local Transfer of Optical Angular Momentum to Matter. I. Mod. Opt. 2005, 52, 1045-1052.

(61) Garcés-Chávez, V.; McGloin, D.; Padgett, M. J.; Dultz, W.; Schmitzer, H.; Dholakia, K. Observation of the Transfer of the Local Angular Momentum Density of a Multiringed Light Beam to an Optically Trapped Particle. Phvs. Rev. Lett. 2003, 91, 093602.

(62) Babiker, M.; Bennett, C. R.; Andrews, D. L.; Dávila Romero, L. C. Orbital Angular Momentum Exchange in the Interaction of Twisted Light with Molecules. Phys. Rev. Lett. 2002, 89, 143601.

(63) Simpson, N. B.; Dholakia, K.; Allen, L.; Padgett, M. J. Mechanical Equivalence of Spin and Orbital Angular Momentum of Light: An Optical Spanner. Opt. Lett. 1997, 22, 52-54.

(64) Tamburini, F.; Mari, E.; Sponselli, A.; Thidé, B.; Bianchini, A.; Romanato, F. Encoding Many Channels on the Same Frequency through Radio Vorticity: First Experimental Test. New J. Phys. 2012, 14, 033001.

(65) Tamburini, F.; Mari, E.; Thidé, B.; Barbieri, C.; Romanato, F. Experimental Verification of Photon Angular Momentum and Vorticity with Radio Techniques. Appl. Phys. Lett. 2011, 99, 204102.

(66) Bauer, C.; Kobiela, G.; Giessen, H. 2d Quasiperiodic Plasmonic Crystals. Sci. Rep. 2012, 2, 681.

(67) Dallapiccola, R.; Gopinath, A.; Stellacci, F.; Dal Negro, L. QuasiPeriodic Distribution of Plasmon Modes in Two-Dimensional Fibonacci Arrays of Metal Nanoparticles. Opt. Express 2008, 16, 5544-5555.

(68) Auguié, B.; Barnes, W. L. Collective Resonances in Gold Nanoparticle Arrays. Phys. Rev. Lett. 2008, 101, 143902.

(69) Matsui, T.; Agrawal, A.; Nahata, A.; Vardeny, Z. V. Transmission Resonances through Aperiodic Arrays of Subwavelength Apertures. Nature 2007, 446, 517-521.
(70) Johnson, P. B.; Christy, R. W. Optical Constants of the Noble Metals. Phys. Rev. B: Condens. Matter Mater. Phys. 1972, 6, 4370-4379. (71) Brown, J. M.; Carrington, A. Rotational Spectroscopy of Diatomic Molecules; Cambridge University Press: Cambridge, UK, 2003.

(72) Herzberg, G.; Huber, K. P. Molecular Spectra and Molecular Structure: IV. Constants of Diatomic Molecules; Van Norstrand Reinhold Co.: New York, 1979.

(73) Herzberg, G. Molecular Spectra and Molecular Structure: III. Electronic Spectra \& Electronic Structure of Polyatomic Molecules; D. Van Nostrtand Co. Inc.: New York, 1966.

(74) Liu, M.; Zentgraf, T.; Liu, Y.; Bartal, G.; Zhang, X. Light-Driven Nanoscale Plasmonic Motors. Nat. Nanotechnol. 2010, 5, 570-573.

(75) Asenjo-Garcia, A.; Manjavacas, A.; García de Abajo, F. J. Stimulated Light Emission and Inelastic Scattering by a Classical Linear System of Rotating Particles. Phys. Rev. Lett. 2011, 106, 213601.

(76) Boriskina, S. V.; Reinhard, B. M. Molding the Flow of Light on the Nanoscale: From Vortex Nanogears to Phase-Operated Plasmonic Machinery. Nanoscale 2012, 4, 76-90.

(77) Boriskina, S. V.; Zheludev, N. I. Singular and Chiral Nanoplasmonics; Pan Stanford: Singapore, 2014.

(78) Proietti Zaccaria, R.; Alabastri, A.; De Angelis, F.; Das, G.; Liberale, C.; Toma, A.; Giugni, A.; Razzari, L.; Malerba, M.; Sun, H. B.; Di Fabrizio, E. Fully Analytical Description of Adiabatic Compression in Dissipative Polaritonic Structures. Phys. Rev. B: Condens. Matter Mater. Phys. 2012, 86, 035410

(79) Schouten, H. F.; Visser, T. D.; Lenstra, D. Optical Vortices near Sub-Wavelength Structures. J. Opt. B: Quantum Semiclassical Opt. 2004, 6, S404.

(80) Padgett, M.; Allen, L. Light with a Twist in Its Tail. Contemp. Phys. 2000, 41, 275-285.

(81) Allen, L.; Beijersbergen, M. W.; Spreeuw, R. J. C.; Woerdman, J. P. Orbital Angular Momentum of Light and the Transformation of Laguerre-Gaussian Laser Modes. Phys. Rev. A: At., Mol., Opt. Phys. 1992, 45, 8185-8189.

(82) Barnett, S. M.; Allen, L. Orbital Angular Momentum and Nonparaxial Light Beams. Opt. Commun. 1994, 110, 670-678.

(83) Alexandrescu, A.; Cojoc, D.; Fabrizio, E. D. Mechanism of Angular Momentum Exchange between Molecules and LaguerreGaussian Beams. Phrs. Rev. Lett. 2006, 96, 243001.

(84) Rury, A. S. Coherent Control of Plasmonic Spectra Using the Orbital Angular Momentum of Light. Phys. Rev. B: Condens. Matter Mater. Phys. 2013, 88, 205132.

(85) Demtroder, W. Atoms, Molecules and Photons; Springer: Heidelberg, 2010.

(86) Eisberg, R.; Resnick, R. Quantum Physics of Atoms, Molecules, Solids, Nuclei, and Particles; John Wiley \& Sons: New York, 1985.

(87) Khristenko, S. V.; Maslov, A. I.; Shevelko, V. P. Molecules and Their Spectroscopic Properties; Springer: Heidelberg, 1998.

(88) Kaufmann, E. N.; Vianden, R. J. The Electric Field Gradient in Noncubic Metals. Rev. Mod. Phvs. 1979, 51, 161-214.

(89) James, H. M.; Coolidge, A. S. Quadrupole Rotation-Vibration Spectrum of $\mathrm{H}_{2}$. Astrophys. I. 1938, 87, 438-459.

(90) Chiu, Y. N. Electric-Quadrupole and Magnetic-Dipole Radiation in Linear Molecules. Applications to $1 \pi-3 \pi$ Transitions. J. Chem. Phys. 1965, 42, 2671-2681.

(91) Halkier, A.; Coriani, S.; Jørgensen, P. The Molecular Electric Quadrupole Moment of $\mathrm{N}_{2}$. Chem. Phvs. Lett. 1998, 294, 292-296.

(92) Li, H.; Le Roy, R. J. Quadrupole Moment Function and Absolute Infrared Quadrupolar Intensities for $\mathrm{N}_{2}$. J. Chem. Phys. 2007, 126, 224301. 